\title{
INFINITESIMAL HOLONOMY GROUPS OF BUNDLE CONNECTIONS
}

\author{
HIDEKI OZEKI
}

\section{Introduction}

In differential geometry of linear connections, A. Nijenhuis has introduced the concepts of local holonomy group and infinitesimal holonomy group and obtained many interesting results [6].

The purpose of the present note is to generalize his results to the case of connections in arbitrary principal fiber bundles with Lie structure groups. The concept of local holonomy group can be immediately generalized and has been already utilized by S. Kobayashi [4]. Our main results are Theorems 4 and 5 on infinitesimal holonomy groups. The proofs depend on a little sharpened form of a theorem of Ambrose-Singer [1]. In the case of linear connections, our infinitesimal holonomy group coincides with that of Nijenhuis, as we shall show in Section 6.

Finally, in the case of Cartan connections, we shall give a certain expression of infinitesimal holonomy groups.

After the completion of this work, we have learned from Dr. A. Nijenhuis that he himself obtained a similar generalization [7].

In preparing the present note for publication, I have received valuable criticism and kind encouragement from Dr. K. Nomizu, Mr. J. Hano and, in particular, from Dr. Nijenhuis. I am glad to express here my deep gratitude to them.

\section{Holonomy groups}

Let $P=P(M, G, \pi)$ be a differentiable principal fiber bundle over a base manifold $M$ with Lie structure group $G$ and with projection $\pi$ of $P$ onto $M$. We always assume that $P$ satisfies the second axiom of countability and that $M$ is connected. By differentiabily, we understand that of class $C^{\infty}$. We denote by

Received December 20, 1955. 
$T_{x}(P)$ the tangent space to $P$ at $x$ and by $V_{x}$ the subspace of $T_{x}(P)$ which is tangent to the fiber through $x$.

A connection in $P$ is given by defining at each point $x$ of $P$ a vector subspace $Q_{x}$ of $T_{x}(P)$ such that
(Q.1)
$Q_{x}$ depends differentiably on $x$;
$(Q .2)$
$T_{x}(P)=V_{x}+Q_{x}($ direct sum $)$;
$(Q .3)$
$Q_{x \cdot a}=R_{a} \cdot Q_{x}$,

where $R_{a}(a \in G)$ denotes the right translation of $P$ induced by $a: R_{a} x=x \cdot a$. A vector in $Q_{x}$ is called horizontal and a vector in $V_{x}$ vertical. Let us denote $h_{x}$ (resp. $v_{x}$ ) the projection of $T_{x}(P)$ onto $Q_{x}$ (resp. $V_{x}$ ) with respect to the direct decomposition (Q.2). For an element $A$ in the Lie algebra $\&$ of $G$, we denote by $A^{*}$ the vertical vector field on $P$ generated by $A$. Here $A^{*}$ generated by $A$ is the vectorfield on $P$ induced by the 1-parameter group $R_{a_{t}}$ of right translations of $P$ where $a_{t}=\exp \cdot t A[1],[8]$. Now define a 9 -valued differential 1 -form $\omega$, called the connection form, on $P$ by
$(\omega .1)$
$(\omega .2)$

$$
\begin{array}{lll}
\omega_{x}\left(A_{x}^{*}\right)=A & \text { for } & A \in g ; \\
\omega_{x}(X)=0 & \text { for } & X \in Q_{x} .
\end{array}
$$

Then $\omega$ satisfies the condition

$$
R_{a}^{*} \cdot \omega=a d\left(a^{-1}\right) \cdot \omega .
$$

Conversely, a g-valued differential 1-form $\omega$ satisfying $(\omega .1)$ and ( $\omega .3)$ defines, by ( $\omega .2)$, a connection $Q_{x}$, whose connection form coincides with $\omega$.

For a given connection, the curvature form $\Omega$ on $P$ is defined by

$$
\Omega_{x}(X, Y)=d \omega_{x}\left(h_{x} \cdot X, h_{x} \cdot Y\right) \quad\left(X, Y \in T_{x}(P)\right) .
$$

$\Omega$ satisfies the condition

$$
R_{a}^{*} \Omega=a d\left(a^{-1}\right) \cdot \Omega .
$$

The structure equation $([1],[8])$ is given by

$$
\Omega=d \omega+\frac{1}{2}[\omega, \omega] .
$$

A g-valued differential form $\theta$ is called of type $\operatorname{ad}(G)$, if it satisfies

$$
R_{a}^{*} \cdot \theta=a d\left(a^{-1}\right) \cdot \theta \quad(a \in G) .
$$


If $\theta$ is of degree 0 , that is. if $\theta$ is a $g$-valued function, this can be written as

$$
\theta(x \cdot a)=a d\left(a^{-1}\right) \cdot \theta(x) .
$$

( $\omega .3$ ) and ( $\Omega .2$ ) show that $\omega$ and $\Omega$ are of type $a d(G)$.

A (piecewise differentiable) curve $x(t)$ in $P$ is called horizontal if, at each point $x(t)$, the tangent vector to the curve is horizontal. We denote by $P(x)$ the set of point $y$ such that $x$ and $y$ can be joined by a horizontal curve in $P$. The holonomy group $H(x)$ at a point $x$ of $P$ is defined by

$$
H(x)=\{a \in G \mid x \cdot a \in P(x)\},
$$

and the restricted holonomy group $H^{0}(x)$ at $x$ is the set of all the elements a of $G$ such that $x$ and $x \cdot a$ can be joined by a horizontal curve whose projection to $M$ is homotopic to 0 in $M$. As is well known [1], [8], $H(x)$ is a Lie subgroup of $G$ and $H^{0}(x)$ coincides with the connected component of $H(x)$ containing the unit element $e$. Denote by $\mathfrak{h}(x)$ the Lie algebra of $H^{0}(x)$, which is a subalgebra of 9 . From these definitions, we get easily

$$
\begin{aligned}
& H(x \cdot a)=a d\left(a^{-1}\right) H(x) \text { and } \mathfrak{h}(x \cdot a)=a d\left(a^{-1}\right) \mathfrak{h}(x) ; \\
& H(y)=H(x) \text { and } \mathfrak{h}(y)=\mathfrak{h}(x) \text { for } y \in P(x) .
\end{aligned}
$$

At each point $x$ of $P$, let $\mathfrak{m}_{0}(x)$ be the vector subspace of 8 spanned by all the elements $\Omega_{x}(X, Y)\left(X, Y \in T_{x}(P)\right)$. Since $\Omega$ is of type $a d(G)$, we have

$$
\mathrm{m}_{0}(x \cdot a)=a d\left(a^{-1}\right) \mathrm{m}_{0}(x) .
$$

Now, the theorem of Ambrose-Singer (Theorem 2 of [1], cf. [5], [8]) states that $\mathfrak{h}(x)$ is the Lie algebra generated by all $m_{0}(y), y \in P(x)$.

Later on, we shall use

Lemma 1. $\mathfrak{h}(x)$ is spanned as a vector subspace in $g$ by all $m_{0}(y), y \in P(x)$.

Proof. Let $\mathrm{m}$ be the vector subspace spanned by all $\mathfrak{n}_{0}(y), y \in P(x)$. For a point $y$ of $P(x)$, let $x(t)$ be a horizontal curve joining $x$ and $y$. Then the curve $x(t) \cdot a$ is also horizontal for any element a of $G$. If $a \in H(x)$, then $x \cdot a \in P(x)$, and hence $y \cdot a \in P(x)$. Therefore we have

$$
a d\left(a^{-1}\right) m_{0}(y)=m_{0}(y \cdot a) \subset m .
$$

This proves $a d\left(a^{-1}\right) \mathfrak{m} \subset \mathrm{m}$, for $a \in H(x)$, and hence $[\mathfrak{h}(x), \mathrm{m}] \subset \mathrm{m}$. This shows that $\mathfrak{m}$ itself forms a Lie algebra. q.e.d. 
Remark. The proofs of Theorems 1 and 4 of Nijenhuis [6] are not sufficient. The proof of Theorem 1 can be completed by the above lemma. But in the statement of Theorem 4, "spanned" has to be replaced by "generated."

\section{Local holonomy groups}

For any connected open subset $U$ of $M, \pi^{-1}(U)$ has the natural bundle structure and the natural connection induced those of $P$. For any point $x$ of $\pi^{-1}(U)$, we denote by $H^{0}(U, x)$ the restricted holonomy group at $x$ of this connection in $\pi^{-1}(U)$ and by $P(U, x)$ the set of points $y$ of $\pi^{-1}(U)$ such that $x$ and $y$ can be joined by a horizontal curve in $\pi^{-1}(U)$.

Definition. The local holonomy group $H^{*}(x)$ at a point $x$ of $P$ is defined as

$$
H^{*}(x)=\bigcap_{p \in U} H^{0}(\tilde{U}, x)
$$

where $U$ is an arbitrary connected open neighborhood of $p=\pi \cdot x$ in $M$.

For a point $x$ of $P$, take a sequence of connected open neighborhoods $U_{k}$ $(k=1,2,3, \ldots)$ of $p=\pi \cdot x$ in $M$ such that $U_{k} \supset \bar{U}_{k+1}$ and $\bigcap_{k} U_{k}=\{p\}$. Then, for any open neighborhood $V$ of $p$, there exists an integer $\alpha$ such that $U$ is contained in $V$ for $k \geqslant \alpha$. Therefore we have $H^{*}(x)=\bigcap_{k} H^{0}\left(U_{k}, x\right)$. Since each $H^{0}\left(U_{k}, x\right)$ is completely determined by its Lie algebra, the minimal values of $\operatorname{dim} H^{0}\left(U_{k}, x\right)$ is attained for some $k$. Hence we have $H^{*}(x)=H^{0}\left(U_{k}, x\right)$ for some $k$.

We have clearly

Proposition 1. The local holonomy group $H^{*}(x)$ has the following properties:

i) $H^{*}(x)$ is a connected Lie subgroup of $G$ which is contained in $H^{0}(x)$. Denote by $\mathfrak{h}^{*}(x)$ the subalgebra associated to $H^{*}(x)$ in $\mathfrak{g}$;

ii) $H^{*}(x \cdot a)=\operatorname{ad}\left(a^{-1}\right) H^{*}(x)$ and $\mathfrak{h}^{*}(x \cdot a)=\operatorname{ad}\left(a^{-1}\right) \mathfrak{h}^{*}(x)$;

iii) There exists a connected open neighborhood $U$ of $p=\pi \cdot x$ such that, for any connected open neighborhood $V$ of $p$ contained in $U$, we have $H^{*}(x)$ $=H^{0}(V, x)$;

iv) For any point $y$ of $P(U, x)$, we have $H^{*}(x) \supset H^{*}(y)$ and $\mathfrak{h}^{*}(x) \supset \mathfrak{h}^{*}(y)$;

v) The set $\left\{\pi(x) \in M \mid \operatorname{dim} H^{*}(x) \leqq \alpha\right\}$ is open in $M$ for any integer $\alpha$. 
As to $\mathrm{v}$ ), we remark that since $\operatorname{dim} H^{*}(x)$ is constant on each fiber, $\operatorname{dim} H^{*}(x)$ can be considered as a function on the base space $M$.

THEOREM 1. $H^{0}(x)$ is generated by all $H^{*}(y), y \in P(x)$, and $\mathfrak{h}(x)$ is spanned by all $\mathfrak{h}^{*}(y), y \in P(x)$.

The first stament means that every element a of $H^{0}(x)$ can be written as a finite product $a=a_{1} \ldots a_{k}$ where $a_{i} \in H^{*}\left(y_{i}\right)$ for some $y_{i} \in P(x)$.

We have $\mathfrak{h}(x)=\mathfrak{h}(y) \supset \mathfrak{h}^{*}(y) \supset \mathrm{m}_{0}(y)$ for a point $y$ of $P(x)$. By Lemma 1 , $\mathfrak{h}(x)$ is spanned by $\mathrm{m}_{0}(y), y \in P(x)$, and a fortiori by $\mathfrak{h}^{*}(y)$. The first stament follows from the second and from the following

Lemma 2. If the Lie algebra a of a connected Lie group $G$ is generated by a family of vector subspaces $\mathfrak{m} \lambda(\lambda \in A)$ of $\mathcal{A}$, then any element $a$ of $G$ can be written as a finite product of the following form

$$
a=\exp X_{1} \cdot \exp X_{2} \ldots \exp X_{k}
$$

where each $X_{i}$ is contained in some $\mathrm{m}_{\lambda}$.

Froof. Let $H$ be the set of those elements a which can be written in the above form. We easily see that $H$ is an arc wise connected subgroup of $G$ and hence a Lie subgroup of $G$ by a theorem of Kuranishi-Yamabe [11]. Since $H$ contains the elements of the form $\exp X, X \in \mathfrak{m}_{\lambda}$, the Lie algebra $\mathfrak{h}$ of $H$ contains each $m_{\lambda}$. From the assumption, $\mathfrak{h}$ coincides with $\mathfrak{A}$.

q.e.d.

THEOREM 2. If $\operatorname{dim} H^{*}(x)$ is constant over $P$, then we have $H^{0}(x)=H^{*}(x)$ for every $x \in P$.

Proof. In a sufficiently small neighborhood $U$ of $p=\pi \cdot x$, we have $H^{*}(x)$ $\supset H^{*}(y)$ for a point $y$ of $P(U, x)$. Since they have the same dimension, we have $H^{*}(x)=H^{*}(y)$. From this, we can easily deduce that for any point $y$ of $P(x)$ we have $H^{*}(x)=H^{*}(y)$. Since $H^{0}(x)$ is generated by all $H^{*}(y)$ for $y \in P(x)$, we have finally $H^{0}(x)=H^{*}(x)$.

\section{Infinitesimal holonomy groups}

The definition of infinitesimal holonomy groups is preceded by several lemmas.

Lemma 3. Let $f$ be a g-valued function of type ad $(G)$ on $P$. Then 
i) For any vector field $X$ on $P$,

$$
v(X)_{x} \cdot f=-\left[\omega_{x}(X), f(x)\right]
$$

ii) For any horizontal vector fields $X$ and $Y$ on $P$,

$$
v([X, Y])_{x} \cdot f=2\left[\Omega_{x}(X, Y), f(x)\right] ;
$$

iii) If $X$ and $Y$ are vector fields on $P$ invariant under right translations, then $\Omega(X, Y)$ and $X \cdot f$ are functions of type ad $(G)$.

Proof. i) Set $A=\omega_{x}(X) \in g$. Then we have $A_{x}^{*}=v(X)_{x}$, and hence

$$
\begin{aligned}
v(X)_{x} \cdot f & =A_{x}^{*} \cdot f=\lim _{t \rightarrow 0} \frac{1}{t}\{f(x \cdot \exp t A)-f(x)\} \\
& =\lim _{t \rightarrow 0} \frac{1}{t}\{\operatorname{ad}(\exp (-t A)) \cdot f(x)-f(x)\} \\
& =-[A, f(x)]=-\left[\omega_{x}(X), f(x)\right]
\end{aligned}
$$

(cf. [2]), where $\exp t A$ is the 1-parameter subgroup of $\mathrm{G}$ generated by $A$.

ii) For any horizontal vector fields $X$ and $Y$, we have, in virtue of the structure equation,

$$
\begin{aligned}
2 \Omega_{x}(X, Y) & =2 d \omega_{x}(X, Y)+\left[\omega_{x}(X), \omega_{x}(Y)\right] \\
& =2 d \omega_{x}(X, Y) \\
& =X_{x}(\omega(Y))-Y_{x}(\omega(X))-\omega_{x}([X, Y]) \\
& =-\omega_{x}([X, Y]) .
\end{aligned}
$$

From this, we get ii) in virtue of i).

iii) Since $\Omega$ is of type $\operatorname{ad}(G)$, we have

$$
\Omega_{x a}\left(R_{a} X, R_{a} Y\right)=\operatorname{ad}\left(a^{-1}\right) \Omega_{x}(X, Y) .
$$

Hence $\Omega(X, Y)$ is a function of type $a d(G)$ if $R_{a} X=X$ and $R_{a} Y=Y$. We have also

$$
\begin{aligned}
(X f)(x a) & =X_{x a} \cdot f=\left(R_{a} X_{x}\right) \cdot f=X_{x}(f \circ R) \\
& =a d\left(a^{-1}\right)\left(X_{x} f\right)=\operatorname{ad}\left(a^{-1}\right)(X f)(x) .
\end{aligned}
$$

We have completed the proof of Lemma 3.

We have defined, in Section $2, m_{0}(x)$ at each point $x$ of $P$ as the vector subspace of $g$ which is spanned by all the elements $\Omega_{x}(X, Y), X, Y \subseteq T_{x}(P)$.

Now we shall define a vector subspace $m_{k}(x)$ of $g$ inductively on $k$ in the following way. Consider any function of the form 
$\left(\mathrm{I}_{k}\right)$

$$
V_{k} \cdot V_{k-1} \ldots V_{1} \Omega(X, Y),
$$

where $X, Y, V_{1}, \ldots, V_{k}$ are arbitrary horizontal vector fields on $P$, and $V_{k} \ldots V_{1} \Omega(X, Y)$ denotes the result of applying successively $V_{1}, \ldots, V_{k}$ to the $g$-valued function $\Omega(X, Y)$. Let $m_{k}(x)$ be the subspace of $g$ spanned by $m_{k-1}(x)$ and by the values at $x$ of all the functions of the form $\left(I_{k}\right)$. Set $f^{\prime}(x)$ $=\bigcup_{k} m_{k}(x)$. We shall later see that this $\mathfrak{h}^{\prime}(x)$ defines the Lie algebra of the infinitesimal holonomy group.

Take a basis $X_{1}, \ldots, X_{n}(n=\operatorname{dim} M)$ of vector fields on a neighborhood $U$ of $p=\pi \cdot x$ in $M$, and let $X_{i}^{*}$ the lift of $X_{i}$ ( to $\pi^{-1}(U)$ ) respectively. Here the lift $X^{*}$ of a vector field $X$ on $M$ is the horizontal vector field on $P$ such that $\pi \cdot X^{*}=X . \quad X^{*}$ is uniquely determined and is invariant by $R_{a}, a \in G$. Consider any function of the form

$$
X_{j_{k}}^{*} \ldots X_{j_{1}}^{*} \Omega\left(X_{i}^{*}, X_{l}^{*}\right)
$$

where $i, l, j_{1}, \ldots, j_{k}$ are taken arbitrarily from $1, \ldots, n$.

Lemma 4. For each $k, m_{k}(x)$ is spanned by $m_{k-1}(x)$ and by the values at $x$ of all the functions of the form $\left(\mathrm{II}_{i}\right)$, furthermore

$$
\mathrm{m}_{k}(x a)=a d\left(a^{-1}\right) \mathrm{m}_{k}(x) .
$$

Proof. The lemma is true for $k=0$, and we use the induction on $k$. Suppose that Lemma 4 is true for $k-1$. From the definition, $m_{k}(x)$ is spanned by $m_{k-1}(x)$ and by the values at $x$ of all the functions of the form $\left(\mathrm{I}_{k}\right)$. Since $X_{1}$, $\ldots, X_{n}^{*}$ form a basis of horizontal vector fields on $\pi^{-1}(U)$, it is easily seen that any function of the form $\left(\mathrm{I}_{k}\right)$ can be written as a linear combination of the functions of the form $\left(\mathrm{II}_{\alpha}\right)(\alpha \leqq k)$, where the coefficients are real valued functions. This shows the first statement of the lemma. Since $X_{i}^{*}$ is right invariant, $\Omega\left(X_{i}^{*}, X_{l}^{*}\right)$ is of type $\operatorname{ad}(G)$. By iii) of Lemma 3 , any function of the form $\left(\mathrm{II}_{k}\right)$ is also of type $a d(G)$. Hence we get $m_{k}(x a)=\operatorname{ad}\left(a^{-1}\right) m_{k}(x)$. q.e.d.

Lemma 5. The vector space $\mathfrak{h}^{\prime}(x)=\bigcup_{k} \mathfrak{m}_{k}(x)$ is contained in $\mathfrak{h}^{*}(x)$.

Proof. We shall prove this by the induction on $k$. For $k=0$, we already know that $m_{0}(x) \subset \mathfrak{h}^{*}(x)$. Suppose that $m_{k-1}(x) \subset \mathfrak{h}^{*}(x)$ for every point $x$. Since $\mathfrak{m}_{k}(x)$ is spanned by $\mathfrak{m}_{k-1}(x)$ and by the values at $x$ of the functions of the form $\left(\mathrm{I}_{k}\right)$, it is sufficient to show that, for any horizontal vector field $X$ and 
for any function $f$ of the form $\left(\mathrm{I}_{k-1}\right)$, we have $X_{x} \cdot f \in \mathfrak{h}^{*}(x)$.

Let $\varphi_{t}$ be the local 1-parameter group of transformations generated by $X$ and defined in a neighborhood of $x$. Then, $\varphi_{t} \cdot x \quad(|t|<$ some $\delta)$ forms a horizontal curve through $x$ since $X$ is horizontal. By iv) of Proposition 1 we have, for sufficiently small $t, \mathfrak{h}^{*}\left(\varphi_{t} \cdot x\right) \subset \mathfrak{h}^{*}(x)$, and hence

$$
f\left(\varphi_{t} \cdot x\right) \in \mathfrak{m}_{k-1}\left(\varphi_{t} \cdot x\right) \subset \mathfrak{h}^{*}\left(\varphi_{t} \cdot x\right) \subset \mathfrak{h}^{*}(x) .
$$

On the other hand, we have

$$
X_{x} \cdot f=\lim _{t \rightarrow 0} \frac{1}{t}\left\{f\left(\varphi_{t} \cdot x\right)-f(x)\right\} .
$$

Therefore we have $X_{x} \cdot f \in \mathfrak{h}^{*}(x)$.

q.e.d.

Lemma 6. $\mathfrak{h}^{\prime}(x)$ spans a subalgebra of $\mathfrak{g}$.

Proof. It is sufficient to show that, for any integers $l$ and $k$, we have $\left[\mathrm{m}_{l}(x), \mathrm{m}_{k}(x)\right] \subset \mathrm{m}_{k+l+2}$. To prove this it is also sufficient to show that, for any function $g$ of the form $\left(\mathrm{I}_{l}\right)$ and $f$ of the form $\left(\mathrm{II}_{k}\right)$, the $g$-valued function $[f, g](x)$ $=[f(x), g(x)]$ can be written as a linear combination of the functions of the form $\left(\mathrm{I}_{r}\right)(r \leqq k+l+2)$ with coefficients of real valued functions. This will be proved by the induction on $l$.

i) In the case where $l=0, g$ has the expression $g(x)=\Omega_{x}(X, Y)$, where $X, Y$ are horizontal vector fields. Since $f$ is of type $a d(G)$, we have, by ii) of Lemma 3,

$$
2\left[\Omega_{x}(X, Y), f(x)\right]=v([X, Y])_{x} \cdot f
$$

On the other hand,

$$
\begin{aligned}
v([X, Y])_{x} \cdot f & =[X, Y]_{x} \cdot f-h([X, Y])_{x} \cdot f \\
& =X_{x}(Y f)-Y_{x}(X f)-h([X, Y])_{x} \cdot f .
\end{aligned}
$$

Since $X \cdot Y \cdot f$ and $Y \cdot X \cdot f$ are of the form $\left(\mathrm{I}_{k+2}\right)$ and $h([X, Y]) \cdot f$ of the form $\left(\mathrm{I}_{k+1}\right)$, the above statement is true for $l=0$ and for any $k$.

ii) Suppose that the statement is true for $l$ and for any $k$. To prove the case $l+1$, it is sufficient to show that, for any function $g$ of the form $\left(I_{l}\right)$ and $f$ of the form $\left(\mathrm{II}_{k}\right)$, the statement holds for the function $[X \cdot g, f]$, where $X$ is any horizontal vector field. We have easily

$$
\left[X_{x} \cdot g, f(x)\right]=X_{x}([g, f])-\left[g(x), X_{x} \cdot f\right] .
$$


Here, the statement holds for $[g ; X \cdot f]$ from the inductive assumption, and the function $[g, f]$ can be written as a linear combination of functions of the form $\left(\mathrm{I}_{r}\right)(r \leqq l+k+2)$ and hence $X([g, f])$ is a linear combination of functions of the form $\left(\mathrm{I}_{r}\right)(r \leqq l+k+3)$. Therefore the statement is also true for $[X g, f]$. This completes the induction.

By Lemma 5 and Lemma $6, \mathfrak{h}^{\prime}(x)$ is a subalgebra of $\mathfrak{h}^{*}(x)$.

Definition. The infinitesimal holonomy group $H^{\prime}(x)$ at a point $x$ of $P$ is the connected Lie subgroup of $G$ which has $\mathfrak{h}^{\prime}(x)$ as its Lie algebra.

PROPOSITION 2. The infinitesimal holonomy group $H^{\prime}(x)$ has the following properties:

i) $H^{\prime}(x)$ is a connected Lie subgroup of $H^{*}(x)$;

ii) $H^{\prime}(x \cdot a)=a d\left(a^{-1}\right) H^{\prime}(x)$ and $\mathfrak{h}^{\prime}(x \cdot a)=a d\left(a^{-1}\right) \mathfrak{h}^{\prime}(x)$;

iii) The set $\left\{\pi \cdot x \in M \mid \operatorname{dim} H^{\prime}(x) \geqq \alpha\right\}$ is open in $M$ for any integer $\alpha$;

iv) If $H^{\prime}(x)=H^{*}(x)$ at a point, then there exists a connected open neighborhood $U$ of $p=\pi \cdot x$ such that, for any point $y$ of $P(U, x)$, we have $H^{\prime}(y)$ $=H^{*}(y)=H^{\prime}(x)=H^{*}(x)$.

Proof. ii) follows from Lemma 4, and it means that $\operatorname{dim} H^{\prime}(x)$ can be considered as a function on $M$. iii) is seen from the fact that if the values of a finite number of functions of the form $\left(\mathrm{I}_{k}\right)(k \geqslant 0)$ are linearly independent at a point $x$, so are they in a neighborhood of $x$. If $\operatorname{dim} H^{\prime}(x)=\operatorname{dim} H^{*}(x)$ at $x$, this holds in a neighborhood of $x$ by $v$ ) of Proposition 1 and by iii) of the above. Hence we get iv) from Theorem 2.

TheOREm 3. $H^{0}(x)$ is generated by all $H^{\prime}(y), y \in P(x)$, and $\mathfrak{h}(x)$ is spanned by all $\mathfrak{h}^{\prime}(y), y \in P(x)$.

The proof is similar to that of Theorem 1.

\section{Theorems}

Theorem 4. If $\operatorname{dim} H^{\prime}(x)$ is constant over $P$, then we have $H^{0}(x)=H^{*}(x)$ $=H^{\prime}(x)$ for every $x \in P$.

This can be obtained from Theorem 2 and from the following

Lemma 7. If $\operatorname{dim} H^{\prime}(x)$ is constant in a neighborhood of $x$ in $P$, then we have $H^{\prime}(x)=H^{*}(x)$. 
Proof. On a sufficiently small neighborhood $V$ of $p=\pi \cdot x$ in $M$, we have, for any point $y$ of $P(V, x)$,

$$
\mathfrak{h}^{*}(x) \supset \mathfrak{h}^{*}(y) \supset \mathfrak{h}^{\prime}(y) \supset \mathfrak{m}_{0}(y) .
$$

Therefore we see from Lemma 1 that $\mathfrak{h}^{*}(x)$ is spanned by all $\mathfrak{h}^{\prime}(y), y \in P(V, x)$. Therefore it is sufficient to show the existence of a neighborhood $U$ of $p$ on which $\mathfrak{h}^{\prime}(x)=\mathfrak{h}^{\prime}(y)$ holds for any $y \in P(U, x)$.

Now take a finite number of functions $f_{1}, \ldots, f_{\alpha}$ of the form $\left(\mathrm{II}_{k}\right)(k \geqslant 0)$ such that $f_{1}(x), \ldots, f_{\alpha}(x)$ form a basis of $\mathfrak{h}^{\prime}(x)$. Then we see from the assumption of the lemma that $f_{1}(y), \ldots, f_{\alpha}(y)$ form a basis of $\mathfrak{h}^{\prime}(y)$ for any point $y$ in a sufficiently small neighborhood of $x$. Since $f_{1}, \ldots, f_{\alpha}$ are of type $a d(G), f_{1}(v a), \ldots, f_{\alpha}(y \cdot a)$ also form a basis of $\mathfrak{h}^{\prime}(y \cdot a)$. This shows that there exists a neighborhood $V$ of $p$ such that $f_{1}(y), \ldots, f_{\alpha}(y)$ form a basis of $\mathfrak{l}^{\prime}(y)$ for every point $y$ of $\pi^{-i}(V)$. Take a neighborhood $U$ of $p$ in $V$ so small that (5.1) holds in $\pi^{-1}(U)$.

We shall prove $\mathfrak{h}^{\prime}(x)=\mathfrak{h}^{\prime}(y)$ for any point $y$ of $P(U, x)$. Let $x(t)$ $(0 \leqq t \leqq 1)$ be a horizontal curve joining $x$ and $y$ in $\pi^{-1}(U)$. Though $x(t)$ is piecewise differentiable in general, we assume first that $x(t)$ is differentiable throughout $0 \leqq t \leqq 1$.

Set $g_{i}(t)=f_{i}(x(t))$. Since the curve $x(t)$ is horizontal, we have

$$
\left(d g_{i} / d t\right)_{t} \in \mathfrak{h}^{\prime}(x(t)) .
$$

As $g_{1}(t), \ldots, g_{\alpha}(t)$ form a basis of $\mathfrak{h}^{\prime}(x(t)), d g_{j} / d t$ can be written as

$$
\left(d g_{j} / d t\right)_{t}=\sum_{j=1}^{\alpha} \alpha_{j k}(t) \cdot g_{k}(t)
$$

where $\alpha_{j k}(t)$ is differentiable in $t$.

Consider the differential equation for the unknown $\beta_{j}$

$$
d \beta_{j} / d t=-\sum_{k=1}^{\alpha} \beta_{k} \cdot \alpha_{k j} \quad(j=1 \ldots \alpha) .
$$

This has $\alpha$-linearly independent solutions $\beta_{i j}$ with initial condition

$$
\beta_{i j}(0)=\delta_{i j}
$$

We see easily that these solutions can be extended for $0 \leqq t \leqq 1$, constructing successively the solutions. We have 


$$
\begin{aligned}
& \frac{d}{d t}\left(\sum_{j=1}^{\alpha} \beta_{i j} \cdot g_{j}\right)=\sum_{j=1}^{\alpha} \frac{d}{d t}\left(\beta_{i j}\right) \cdot g_{j}+\sum_{j=1}^{\alpha} \beta_{i j} \cdot \frac{d}{d t} g_{j} \\
& =-\sum_{j=1}^{\alpha} \sum_{k=1}^{\alpha} \beta_{i k} \cdot \alpha_{k j} \cdot g_{j}+\sum_{j=1}^{\alpha} \sum_{k=1}^{\alpha} \beta_{i j} \cdot \alpha_{j k} \cdot g_{k}=0 .
\end{aligned}
$$

Hence we have $\sum_{j=1}^{\alpha} \beta_{i j}(t) \cdot g_{j}(t)=g_{i}(0)$. This proves $\mathfrak{h}^{\prime}(x(0))=\mathfrak{h}^{\prime}(x(1))$, because the $\operatorname{det}\left|\beta_{i j}(1)\right|$ is not 0 . This also can be proved successively for the case where $x(t)$ is piece-wise differentiable. Therefore we have proved $H^{\prime}(x)=H^{*}(x)$.

A point $p$ of $M$ is called singular with respect to $H^{*}$ ( resp. $H^{\prime}$ ) if, for any sufficiently small neighborhood $U$ of $p, \operatorname{dim} H^{*}(x)$ (resp. $\operatorname{dim} H^{\prime}(x)$ ) is not constant over $\pi^{-1}(U)$. From Lemma 7 and from iv) of Proposition 2, we see that the set $\Omega^{*}$ of singular points of $H^{*}$ is contained in the set $\Omega^{\prime}$ of singular points of $H^{\prime}$. In the same way as Nijenhuis [6], we can prove that $\Omega^{*}$ and $\Omega^{\prime}$ are closed sets without interior points.

In the case of an analytic bundle, a connection is called analytic if the horizontal space $Q_{x}$ depends analytically on $x$. Then one can see that the horizontal component $h X$ of any analytic vector field $X$ is analytic and that $\omega$ and $\Omega$ are also analytic.

THEOREM 5. If the bundle and the connection are analytic, then, $H^{0}(x)$ $=H^{*}(x)=H^{\prime}(x)$ for every $x \in P$.

Proof. It is sufficient to prove that $\operatorname{dim} H^{\prime}(x)$ is locally constant. In fact, in this case, $\operatorname{dim} H^{\prime}(x)$ is constant over $P$ and hence we get the theorem from Theorem 4.

Now take analytic local coordinates $x, \ldots, x^{n}$ defined in a neighborhood $U$ of $p=\pi \cdot x$ in $M$. We can assume that $x^{i}(p)=0$ and that $U$ is homeomorphic with the open subset of $R^{n}$ composed of the points $\left(x^{1}, \ldots, x^{n}\right)$ such that $\sum_{i=1}^{n}\left(x^{i}\right)^{2}<a^{2}(a>0)$, by the mapping $q \rightarrow\left(x^{1}(q), \ldots, x^{n}(q)\right)$. We shall prove that $\operatorname{dim} \mathfrak{h}^{\prime}(y)$ is constant over $\pi^{-1}(U)$.

Set $X_{i}=\partial / \partial x^{i}$ and let us denote by $X_{i}^{*}$ the lift of $X_{i}$. For any system of $n$-real numbers $\left(a^{1}, \ldots, a^{\prime \prime}\right)$ such that $\sum_{i=1}^{n}\left(a^{i}\right)^{2}=1$. Consider the vector field $X=\sum_{i=1}^{n} a^{i} X_{i}$ on $U$. The ray $u(t)(-a<t<a)$ defined by $x^{i}(u(t))=a^{i} t$ has $X_{\boldsymbol{u}(t)}$ as its tangent vector at $u(t)$. The lift $X^{*}$ of $X$ is given by $\sum_{i=1}^{n} a^{i} X_{i}^{*}$ and is an analytic horizontal vector field on $\pi^{-1}(U)$. The lift $x(t)$ of $u(t)$ through $x$ has $X_{x(t)}^{*}$ as its tangent vector at $x(t)$. Since the set of all the lifts $x(t)$ 
of any ray $u(t)$ defined in the above way covers $U$, it is sufficient to show $\mathfrak{h}^{\prime}(x(0))=\mathfrak{h}^{\prime}\left(x\left(t_{1}\right)\right)$ for any point $x\left(t_{1}\right)$ on the curve $x(t)$.

Now consider all the functions $f$ of the form $\left(\mathrm{II}_{k}\right)(k \geqslant 0)$

$$
f=X_{j_{k}}^{*} \ldots X_{j_{1}}^{*} \Omega\left(X_{i}^{*}, X_{l}^{*}\right)
$$

defined on $\pi^{-1}(U)$, and set $g(t)=f(x(t))$, which are analytic functions defined for $-a<t<a$. Fix a point $x\left(t_{0}\right)$. We can prove (see the lemma below) that there exists $\delta>0$ such that all the functions $g(t)$ so obtained can be expanded in the common neighborhood $\left|t-t_{0}\right|<\delta$ of $t_{0}$ as follows

$$
\begin{aligned}
& g(t)=\sum_{m} \frac{\left(t-t_{0}\right)^{m}}{m !} g^{(m)}\left(t_{0}\right) \\
& g\left(t_{0}\right)=\sum_{m} \frac{\left(t_{0}-t\right)^{m}}{m !} g^{(m)}(t),
\end{aligned}
$$

where we have

$$
\begin{aligned}
& g^{\prime}(t)=X_{x(t)}^{*} \cdot f \\
& g^{\prime \prime}(t)=X_{x(t)}^{*}\left(X^{*} f\right)
\end{aligned}
$$

Therefore, for each $t$ in $\left|t-t_{0}\right|<\delta$, all $g^{(m)}(t)$ belong to $\mathfrak{h}^{\prime}(x(t))$. Since all $g(t)$ span $\mathfrak{h}^{\prime}(x(t))$, the first Taylor expansion shows that $\mathfrak{h}^{\prime}(x(t))$ is contained in $\mathfrak{h}^{\prime}\left(x\left(t_{0}\right)\right)$. Similarly, $\mathfrak{h}^{\prime}\left(x\left(t_{0}\right)\right) \subset \mathfrak{h}^{\prime}(x(t))$ and hence they coincide. For each $t_{1}\left(\left|t_{1}\right|<a\right)$, the set $\left\{x(t)|0 \leqq| t|\leqq| t_{1} \mid\right\}$ being compact, we have $\mathfrak{h}^{\prime}(x(0))$ $=\mathfrak{h}^{\prime}\left(x\left(t_{1}\right)\right)$.

q.e.d.

LEMMA 8. In an analytic manifold, let $x(t)(|t|<a, x(0)=p)$ be the integral curve of an analytic vector field $X$ which is defined in a neighborhood $V$ of $p$ and is not 0 everywhere. For an analytic function $f$ and for a finite number of analytic vector fields $X_{1}, \ldots, X_{\alpha}$ defined on $V$, consider all the functions $g(x)$ and $h(t)$ of the form

$$
\begin{aligned}
& g(x)=\left(X_{j_{k}} \ldots X_{j_{1}} f\right)(x) \\
& h(t)=g(x(t)),
\end{aligned}
$$

where $j_{1}, \ldots, j_{k}$ are arbitrarily taken from $1, \ldots, \alpha$. Then there exists $\delta>0$ such that all $h(t)$ so obtained can be expanded in the common neighborhood $|t|<\delta$ as follows

$$
h(t)=\sum_{m} \frac{t^{m}}{m !} h^{(m)}(0)
$$


Proof. From the assumption, we can take analytic local coordinates ( $x^{1}$, $\left.\ldots, x^{n}\right)$ such that the curve $(t, 0, \ldots, 0)\left(|t|<\right.$ some $\left.a_{1}\right)$ coincides with $x(t)$ and $X=\partial / \partial x^{1}$ (see $\S$ VII, Chap. III of [2]). Now we see that the above expansion of $h(t)$ is the same as that of $g(x)$ in a power sieries of $x^{1}$. Each $X_{i}$ can be

written as $X_{i}=\sum_{j=1}^{n} f_{i j} \partial / \partial x^{i}$. Since $f$ and $f_{i j}$ are analytic, they can be expanded in a power series of $\left(x^{1}, \ldots, x^{n}\right)$ in a common neighborhood $\left|x^{i}\right|<a_{2}$. Now the lemma can be seen from the following fact:

If two analytic functions $f_{1}, f_{2}$ of $\left(x^{1}, \ldots, x^{n}\right)$ are expanded in a neighborhood $\left|x^{i}\right|<a$, then the functions $f_{1} \cdot f_{2}$ and $f_{1} \cdot \partial f_{2} / \partial x^{i}$ can be expanded in the same neighborhood.

q.e.d.

\section{Linear connections}

Let $P$ be the bundle of frames over a connected $n$-dimensional manifold $M$. $P$ is a principal fiber bundle with structure group $G L(n, R)$ over $M$. A connection in $P$ is called a linear connection of $M$. The purpose of this section is to show that our infinitesimal holonomy group coincides with that of Nijenhuis. In order to do this, we must first clarify the relation between a connection in $P$ and the covariant differentiation on $M$ induced by this linear connection. We shall follow the formulation which has been given recently by $\mathrm{K}$. Nomizu [8].

A connection in $P$ induces a covariant differentiation of tensor fields on $M$ [8], [9]. Let us denote by $\nabla_{X} T$ the covariant differential of a tensor field $T$ on $M$ in the direction of a vector field $X$. Let $F$ be an $n$-dimensional vector space with fixed basis $\xi_{1}, \ldots, \xi_{n}$. Since $P$ is the associated principal bundle of the tangent bundle of $M$, any point $x=\left(e_{1}, \ldots, e_{n}\right)$ of $P$ gives a linear mapping of $F$ noto the tangent space $T_{p}(M)$ to $M$ at $p=\pi \cdot x$ such that

$$
x: \xi_{i} \rightarrow e_{i} .
$$

Since $\operatorname{gr}(n, R)$ is considered as the Lie algebra of all linear endomorphisms of $F$, each element $A$ of $\mathfrak{g l}(n, R)$ gives, for each point $x$ of $P$, a linear endomorphism of $T_{p}(M)(p=\pi \cdot x)$ of the form

$$
x \cdot A \cdot x^{-1}: T_{p}(M) \rightarrow T_{p}(M) .
$$

In the following, we shall denote by $X^{*}$ the lift of a vector field $X$ on $M$ and by $X, Y, Z, V_{1}, \ldots, V_{k}, \ldots$ arbitrary vector fields on $M$.

We shall use the following (see K. Nomizu [8]) 
Lemma 9. For each $Z$, define an F-valued function $f_{Z}$ on $P$ by

$$
f_{Z}(x)=x^{-1}\left(Z_{p}\right) \text {, where } p=\pi \cdot x .
$$

Then we have

$$
\left(\nabla_{X} Z\right)_{p}=x\left(X_{x}^{*} \cdot f\right)
$$

And the curvature tensor $R$ on $M$ is given by

$$
\begin{aligned}
R(X, Y)(Z)_{p} & =\left\{x, \Omega_{x}\left(X^{*}, Y^{*}\right) \cdot x^{-1}\right\}\left(Z_{p}\right) \\
& =x\left\{\Omega_{x}\left(X^{*}, Y^{*}\right)\left(f_{Z}(x)\right)\right\} .
\end{aligned}
$$

A tensor field $T$ of type (1.1) is a rule which assigns to each $p \in M$ a linear endomorphism of $T_{p}(M)$. The covariant differential $\nabla_{V} T$ of $T$ is given by $([8],[9])$

$$
\left(\nabla_{V} T\right)(Z)=\nabla_{V}(T(Z))-T\left(\nabla_{V} \cdot Z\right)
$$

In particular, if $T=R(X, Y)(X, Y$ fixed $)$, we have

$$
\left(\nabla_{V} R(X, Y)\right)(Z)=\nabla_{V}(R(X, Y)(Z))-R(X, Y)\left(\nabla_{V} Z\right)
$$

for any $Z$. The covariant differentiatial of $\nabla_{V_{1}} R(X, Y)$ taken in the directions $V_{2}, \ldots, V_{k}$ successively will be denoted by

$$
\nabla_{V_{2}} \nabla_{V_{1}} R(X, Y), \ldots, \quad \nabla_{V_{k}} \ldots \nabla_{V_{1}} R(X, Y) \text {. }
$$

Lemma $10 . \quad\left(\nabla_{V_{k}} \ldots \nabla_{V_{1}} R(X, Y)\right)(Z)_{p}$

$$
=\left\{x \cdot\left(V_{k}^{*}\right)_{x}\left(V_{k-1}^{*} \ldots V_{1}^{*} \Omega\left(X^{*}, Y^{*}\right) \cdot x^{-1}\right\}\left(Z_{p}\right) \quad(p=\pi \cdot x) .\right.
$$

Proof. The case where $k=0$ is just (6.2). For a vector field $W=R(X, Y)(Z)$, we have

$$
f_{W}(x)=x^{-1}\left\{R(X, Y)(Z)_{p}\right\}=\Omega_{x}\left(X^{*}, Y^{*}\right)\left(f_{Z}(x)\right) .
$$

By (6.1) and (6.5), we get

$$
\left.\left\{\nabla_{V_{1}}(R(X, Y)(Z))\right\}_{p}=x \cdot\left\{V_{1 x}^{*} \Omega\left(X^{*}, Y^{*}\right)\left(f_{Z}\right)\right)\right\}
$$

where $\Omega\left(X^{*}, Y^{*}\right)\left(f_{Z}\right)$ is considered as an $F$-valued function on $P$. On the other hand, we have

$$
\begin{aligned}
& \left.V_{1 x}^{*} \Omega\left(X^{*}, Y^{*}\right)\left(f_{Z}\right)\right) \\
& \quad=\left\{V_{1 x}^{*} \cdot \Omega\left(X^{*}, Y^{*}\right)\right\}\left(f_{2}(x)\right)+\Omega_{x}\left(X^{*}, Y^{*}\right)\left(V_{1 x}^{*} \cdot f_{Z}\right) .
\end{aligned}
$$

From (6.1), this can be written as 


$$
\begin{aligned}
& \left\{V_{1 x}^{*} \cdot \Omega\left(X^{*}, Y^{*}\right)\right\}\left(x^{-1}\left(Z_{p}\right)\right) \\
& \quad=V_{1 x}^{*}\left(\Omega\left(X^{*}, Y^{*}\right)\left(f_{Z}\right)\right)-\Omega_{x}\left(X^{*}, Y^{*}\right)\left(x^{-1}\left(\left(\Gamma_{r_{1}} Z\right)_{p}\right)\right)
\end{aligned}
$$

From (6.4) and (6.5), we get

$$
\left(\nabla_{r_{1}} R\left(X, Y^{\prime}\right)\right)(Z)_{p}=\left\{x \cdot V_{1 x}^{*} \Omega\left(X^{*}, Y^{*}\right) \cdot x^{-1}\right\}\left(Z_{p}\right) .
$$

This proves the lemma for the case $k=1$. The general case can be obtained successively by using (6.3) for $T=\nabla_{1 !-1} \ldots \Gamma_{r_{1}} R(X, Y)$, in stead of (6.4) in the above argument.

The holonomy group $H(x)$ (resp. $H^{*}(x), H^{\prime}(x)$ ) can be represented on $T_{p}(M)(p=\pi \cdot x)$ as the group of linear automorphisms $\left\{x \cdot a \cdot x^{-1} \mid a \in H(x)\right.$ (resp. $\left.\left.H^{*}(x), H^{\prime}(x)\right)\right\}$. Then $\mathfrak{h}(x)$ (resp. $\mathfrak{h}^{*}(x), \mathfrak{l}^{\prime}(x)$ ) is represented on $T_{p}(M)$ as $x \cdot \mathfrak{h}(x) \cdot x^{-1}\left(\operatorname{resp} . x \cdot \mathfrak{h}^{*}(x) \cdot x^{-1}, x \cdot \mathfrak{h}^{\prime}(x) \cdot x^{-1}\right)$. From Lemmas 4 and 10 , we get the following

Lemma 11. $x \cdot \mathfrak{h}^{\prime}(x) \cdot x^{-1}$ is spanned by the values at $p=\pi \cdot x$ of all the tensors of the form

$$
R(X, Y), \nabla_{r_{1}} R(X, Y), \ldots, \quad \nabla_{r^{\prime}} \ldots \nabla_{r_{1}} R(X, Y), \ldots .
$$

THEOREM 6. The Lie algebra $x \cdot \mathfrak{h}^{\prime}(x) \cdot x^{-1}$ of the infinitesimal holonomy group represented on the tangent space $T_{p}(M)(p=\pi \cdot x)$ is spanned by the values at $p$ of all the tensors of the form

$$
R(X, Y),(\nabla R)\left(V_{1} ; X, Y\right), \ldots,\left(\nabla^{(k)} R\right)\left(V_{1}, \ldots, V_{k} ; X, Y\right), \ldots,
$$

where $X, Y, V_{1}, \ldots, V_{k}, \ldots$ are arbitrary vector fields on $M$.

The successive covariant differentiatials $\nabla R, \ldots, \nabla^{(k)} R$ of $R$ can be expressed as follows:

$$
\begin{aligned}
& (\nabla R)\left(V_{1} ; X, Y\right)=\nabla_{V_{1}} \cdot R(X, Y)-R\left(\nabla_{r_{1}} X, Y\right)-R\left(X, \nabla_{r_{1}} Y\right) \\
& \left(\nabla^{(2)} R\right)\left(V_{1}, V_{2} ; X, Y\right)=\nabla_{r_{2}} \cdot\left(\nabla R\left(V_{1} ; X, Y\right)\right) \\
& \quad-\nabla R\left(\nabla_{r_{2}} \cdot V_{1} ; X, Y\right)-\nabla R\left(V_{1} ; \nabla_{r_{2}} X, Y\right)-R\left(V_{1} ; X, \nabla_{r_{2}} Y\right) \\
& \quad \ldots .
\end{aligned}
$$

From (6.10), and from Lemma 11, we obtain the theorem.

Remark. In coordinates $\left(x^{1}, \ldots, x^{n}\right)$, set $X_{i}=\partial / \partial x^{i}$. Then $\nabla_{x i} X_{j}$ can be expressed as 


$$
\nabla_{X i} X_{i}=\sum_{k=1}^{n} \Gamma_{i j}^{k} X_{k}
$$

$\left(\nabla^{(k)} R\right)\left(V_{1}, \ldots, V_{k} ; X, Y\right)$ is expressed as follows :

$$
\left(\nabla^{(k)} R\right)\left(X_{j_{1}}, \ldots, X_{j_{k}} ; X_{i}, X_{l}\right)=\sum_{\alpha, \beta} \nabla_{j_{k} \ldots j_{1}} R_{i i_{\beta}}{ }^{\alpha} \cdot d x^{\beta} \otimes X_{\alpha} .
$$

Theorem 6 is nothing but Theorem 5 of Nijenhuis [6].

\section{Cartan connections}

In this section, we shall show how the infinitesimal holonomy group of a Cartan connection can be expressed on the reduced bundle. However, we do not give here in detail the notion of a Cartan connection (see Ehresmann [3], Kobayashi [5]).

The structure group of a differentiable principal fiber bundle $P=P(M, G)$ is said to be reducible to a Lie subgroup $G^{\prime}$ of $G$ if there exists a differentiable principal fiber bundle $P^{\prime}=P^{\prime}\left(M, G^{\prime}\right)$ with structure group $G^{\prime}$ and a differentiable mapping $\varphi$ of $P^{\prime}$ into $P$ such that $\varphi(x \cdot a)=\varphi(x) \cdot a\left(a \in G^{\prime}, x \in P^{\prime}\right)$ and $\varphi$ is base-preserving [10]. In this case, $P^{\prime}$ with together $\varphi$ is called the reduced bundle and $P^{\prime}$ can be considered as a submanifold of $P$ (so that we consider the set $P^{\prime}$ as a subset of $P$ by the injection $\varphi$ ).

We shall consider in this section only the case where the structure group of $P$ is reducible to a closed subgroup $G^{\prime}$ of $G$ such that $\operatorname{dim} G / G^{\prime}=\operatorname{dim} M$, and we denote by $\left(P^{\prime}, \varphi\right)$ the reduced bundle. Then a connection on $P$ is called Cartan connection if the restriction $\omega^{\prime}=\varphi^{*} \cdot \omega$ of its connection form $\omega$ to $P^{\prime}$ satisfies the condition:

If $\omega_{x}^{\prime}(X)=0\left(X \in T_{x}\left(P^{\prime}\right)\right)$, then $X=0$.

Such a connection exists if and only if the associated bundle $E\left(M, F, G^{\prime}\right)$ of $P$ is "soude à $M$," where $F=G / G^{\prime}$ and $G^{\prime}$ acts on $F$ to the left [3], [5].

For a Cartan connection, let us denote by $\Omega^{\prime}$ the restriction $\varphi^{*} \cdot \Omega$ of the curvature form $\Omega$ to $P^{\prime}$.

Take a subspace $m$ of $\mathfrak{g}$ such that $\mathfrak{g}=\mathfrak{g}^{\prime}=\mathfrak{m}$ (direct sum as vector spaces) where $g^{\prime}$ is the subalgebra corresponding to the identity component of $G^{\prime}$. For any vector field $X$ on $M$ we are denoting by $X^{*}$ the lift of $X$ to $P$. We shall also denote by $X^{\prime}$ the vector field on $P^{\prime}$ such that, at each point $x$ of $P^{\prime}$, $\omega_{x}^{\prime}\left(X_{x}^{\prime}\right) \in m$ and $\pi_{x}\left(X_{x}^{\prime}\right)=X_{\pi \cdot x} . \quad X^{\prime}$ is uniquely determined, because $\pi_{x}$ is a 
linear isomorphism of the vector space $\left\{Y \in T_{x}\left(P^{\prime}\right) \mid \omega_{x}^{\prime}(Y) \in \mathfrak{m}\right\}$ onto $T_{\pi \cdot x}(M)$.

Lemma 12. Let $f$ be a g-valued function on $P$ of type ad $(G)$. Then, for any vector field $V$ on $M$, the function $V^{*} f$ is also of type ad $(G)$, and its restriction to $P^{\prime}$ is equal to the function

$$
V^{\prime} \cdot f^{\prime}+\left[\omega^{\prime}\left(V^{\prime}\right), f^{\prime}\right]
$$

where $f^{\prime}$ denotes the restriction of $f$ to $P^{\prime}$.

Proof. Since $V^{*}$ is right invariant on $P, V^{*} f$ is of type $\operatorname{ad}(G)$ by iii) of Lemma 3. At any point $x$ of $P^{\prime}$, we have

$$
V_{x}^{*}=h_{x} \cdot V_{x}^{*}=h_{x} \cdot V_{x}^{\prime}
$$

because $\pi_{x} \cdot V_{x}^{*}=\pi_{x} \cdot V_{x}^{\prime}=V_{\pi x}$. Hence the vector $\tilde{V}_{x}=V_{x}^{\prime}-V_{x}^{*}$ is vertical in $P$. By i) of Lemma 3 , we have

$$
\tilde{V}_{x} \cdot f=-\left[\omega_{x}\left(\tilde{V}_{x}\right), f(x)\right]
$$

Since $V_{x}^{*}$ is horizontal and since $V_{x}^{\prime}$ is tangent to $P^{\prime}$, we have

$$
\omega_{x}\left(\widetilde{V}_{x}\right)=\omega_{x}\left(V_{x}^{\prime}\right)=\omega_{x}^{\prime}\left(V_{x}^{\prime}\right) .
$$

Therefore, at each point $x$ of $P^{\prime}$,

$$
\begin{aligned}
V_{x}^{*} \cdot f & =V_{x}^{\prime} \cdot f-\widetilde{V}_{x} \cdot f \\
& =V_{x}^{\prime} \cdot f^{\prime}+\left[\omega_{x}\left(\widetilde{V}_{x}\right), f(x)\right] \\
& =V_{x}^{\prime} \cdot f^{\prime}+\left[\omega_{x}^{\prime}\left(V_{x}^{\prime}\right), f^{\prime}(x)\right]
\end{aligned}
$$

This proves our lemma.

Using the above notation, $\mathfrak{h}^{\prime}(x)$ of a Cartan connection can be calculated on the reduced bundle from the following

THEOREM 7. The Lie algebra $\mathfrak{h}^{\prime}(x)$ of the infinitesimal holonomy group of a Cartan connection at a point $x$ of the reduced bundle $P^{\prime}$ is spanned by the values at $x$ of all the functions $f^{(k)}$ on $P^{\prime}$ obtained in the following way:

For any vector fields $X, Y, V_{1}, \ldots, V_{k}, \ldots$ on $M$, define s-valued func. tions $f^{(k)}$ on $P^{\prime}$ successively by

$$
\begin{aligned}
f^{(0)} & =\Omega^{\prime}\left(X^{\prime}, Y^{\prime}\right) \\
f^{(1)} & =V_{1}^{\prime} \cdot f^{(0)}+\left[\omega^{\prime}\left(V_{1}^{\prime}\right), f^{(0)}\right] \\
& =V_{1}^{\prime} \Omega^{\prime}\left(X^{\prime}, Y^{\prime}\right)\left[\omega^{\prime}\left(V_{1}^{\prime}\right), \Omega^{\prime}\left(X^{\prime}, Y^{\prime}\right)\right] \ldots \\
f^{(k+1)} & =V_{k+1}^{\prime} \cdot f^{(k)}+\left[w^{\prime}\left(V_{k+1}^{\prime}\right), f^{(k)}\right] \ldots
\end{aligned}
$$


Proof. Remark first that, at each point $x$ of $P^{\prime}$, we have

$$
\Omega_{x}^{\prime}\left(X^{\prime}, Y^{\prime}\right)=\Omega_{x}\left(X^{*}, Y^{*}\right)
$$

since $h_{x} \cdot X_{\mathrm{r}}^{\prime}=h_{x} \cdot X_{\mathrm{r}}^{*}, h_{x} \cdot Y_{\mathrm{r}}^{\prime}=h_{x} \cdot Y_{x}^{*}$. This shows that $f^{(0)}=\Omega^{\prime}\left(X^{\prime}, Y^{\prime}\right)$ is just the restriction to $P^{\prime}$ of the function $\Omega\left(X^{*}, Y^{*}\right)$. Applying Lemma 12 to the function $\Omega\left(X^{*}, Y^{*}\right)$, we see that $f^{(1)}$ is aiso the restriction to $P^{\prime}$ of $V_{1}^{*} \Omega\left(X^{*}, Y^{*}\right)$. In the same way, we see that $f^{(k)}$ is the restriction to $P^{\prime}$ of $V_{k}^{*} \ldots V_{1}^{*} \Omega\left(X^{*}, Y^{*}\right)$ which is a $g$-valued function on $P$ of type $a d(G)$. This proves the Theorem.

Remark. In general, the vector field $X^{\prime}$ is not right invariant (for instance, in the case of conformal connections). However, from the above proof we see that the function $f^{(k)}$ defined as above are independent on the choice of $\mathrm{m}$ such that $\mathfrak{g}=g^{\prime}+m$.

In particular, in the case of affine connections, the generators of the nonhomogeneous holonomy groups in the above theorem can be given by certain cxpressions involving the torsion and curvature tensors on the base manifold. We hope to take up this problem at another occasion.

\section{BIBLIOGRAPHY}

[1] W. Ambrose and I. Singer, A theorem on holonomy, Trans. Amer. Math. Soc., 75 (1953), 428-443.

[2] C. Chevalley, Theory of Lie groups I, Princeton 1946.

[3] C. Ehresmann, Les connexions infinitésimales dans un espace fibré differentiable, Colloque de Topologie, Bruxelles (1950), 29-55.

[4] S. Kobayashi, Holonomy groups of hyper surfaces, in this Journal.

[5] S. Kobayashi, Theory of connections, part I, Mimeographed notes, University of Washington, Seattle, 1955.

[6] A. Nijenhuis, On the holonomy groups of linear connections, Koninkl. Nederl. Akademie van Wetenschappen, Amsterdam, Proc., Series A, 56 (1953), 233-249. A, 57 (1954), 17-25.

[ 7 ] A. Nijenhuis, Abstract, No. 334 t, Bull. Amer. Math. Soc. vol. 61, (1955), p. 169.

[8] K. Nomizu, Lie groups and Differential Geometry, No. 2, Publications Math. Soc. Japan, to appear shortly.

[9] K. Nomizu, Invariant affine connections on homogeneous spaces, Amer. Jour. Math., 76 (1954), 33-65.

[10] K. Nomizu, Reduction theorem for connections and its applications to the problem 
of isotropy and holonomy groups of Riemannion manifold, Nagoya Math. Jour. 9 (1955), 57-66.

[11] H. Yamabe, On arcwise connected subgroups of a Lie group, Osaka Math. Jour. 2 (1950), 13-14.

\section{Mathematical Institute}

Nagoya University 